PoS $\quad \begin{aligned} & \text { PROCEEDINGS } \\ & \text { OF SCIENCE }\end{aligned}$

\title{
Measurements of the underlying event activity and double parton scattering processes using the CMS detector
}

\author{
Ankita Mehta* \\ (On behalf of the CMS Collaboration) \\ Panjab University Chandigarh (India) \\ E-mail: ankita.mehta@cern.ch
}

Recent results on the double parton scattering studies and measurement of the underlying event activity, performed using proton-proton collisions data collected using the CMS detector are presented.

EPS-HEP 2017, European Physical Society conference on High Energy Physics 5-12 July 2017

Venice, Italy

${ }^{*}$ Speaker. 


\section{Introduction}

The underlying event (UE) activity is a very crucial ingredient to study hadronic collisions and for the tuning of Monte-Carlo (MC) simulations. The UE activity is represented as everything that happens in a collision, except the hard scattering. The UE activity gets the contributions from the beam-beam remnants and the multiple-parton interactions (MPI). The observables used to study the UE activity also receive contributions from the initial-and final-state radiations, making it difficult to uniquely identify the various components of the scattering. Experimental measurement of the UE activity is performed using a process that defines the scale of the hard interaction, along with a phase space region characterized with enhanced particle production from the UE activity. In general, the UE activity leads to the production particles with low-transverse momenta $\left(\mathrm{p}_{\mathrm{T}}\right)$ but there is possibility for the production of high- $\mathrm{p}_{\mathrm{T}}$ particles. Events leading to the production of high- $\mathrm{p}_{\mathrm{T}}$ particles from two independent parton-parton interactions, within a single proton-proton (pp) collision are classified as double parton scattering (DPS). The production cross section for a DPS process is quantified in terms of the effective cross section $\left(\sigma_{\text {eff }}\right)$ which reflects the amount of transverse overlap between the spatial distributions of the colliding protons. Thus, the studies of UE activity and DPS provide a valuable insight into the nature of particle production and correlation among them. Recent measurements of the UE activity and DPS cross section, performed based on the pp collisions data collected using the CMS detector [1] at a center-of-mass energy of $\sqrt{\mathrm{s}}=13$ $\mathrm{TeV}$, are reported in this note.

\section{Underlying event activity}

The measurements of UE activity based on events with leading object either a $\mathrm{Z}$ boson or $t \bar{t}$ pairs are presented. The decay of $\mathrm{Z}$ boson is considered into a pair of muons, while the $t \bar{t}$ events lead to a muon+4jets final state. The UE activity is quantified using observables such as charged particle multiplicity $\left(\mathrm{N}_{\mathrm{ch}}\right)$, scalar sum of $\mathrm{p}_{\mathrm{T}}$ of the charged particles $\left(\sum \mathrm{p}_{\mathrm{T}}\right.$ or $\left.\sum \mathrm{p}_{\mathrm{T}}^{\mathrm{ch}}\right)$, etc. Different phase space regions are defined based on the azimuthal angular separation $(\Delta \phi)$ between a charged particle and the leading object of the event. The transverse regions $\left(60^{\circ}<|\Delta \phi|<120^{\circ}\right)$ are sensitive to the MPI, radiative contributions and modelling of the UE activity, while the recoil activity dominates in the away region $\left(|\Delta \phi|>120^{\circ}\right)$ as compared to the towards $\left(|\Delta \phi|<60^{\circ}\right)$ and transverse regions.

For the measurement of UE activity using $\mathrm{Z}$ boson events, the stable-particle level data distribution of the particle density and energy density $\left(\mathrm{N}_{\mathrm{ch}}\right.$ and $\sum \mathrm{p}_{\mathrm{T}}$ averaged over $\Delta \phi$ and pseudorapidity separation $(\Delta \eta)$ between a charged particle and the dimuon system) are compared with different predictions from simulation [2]. Figure 1 (left) shows the comparison of UE activity measured for the $\mathrm{Z}$ boson events at different center-of-mass energies and predictions from POWHEG+PYTHIA 8 and POWHEG+HERWIG++ event generators. The POWHEG+PYTHIA 8 predictions describe the measurements within 5-10\%, while the POWHEG+HERWIG++ describes the data at different levels of agreement for different values of $\sqrt{\mathrm{s}}$. The mass of $\mathrm{Z}$ boson sets the scale of hard interaction and thus leads to a maximal MPI contributions in such events. For small values of $\mathrm{p}_{\mathrm{T}}$ of the dimuon system $\left(\mathrm{p}_{\mathrm{T}}^{\mu \mu}\right)$, increase in the UE activity with $\mathrm{p}_{\mathrm{T}}^{\mu \mu}$ results in minimal radiative contributions as 
shown in Figure 1 (right). The logarithmic increase in UE activity with $\sqrt{\mathrm{s}}$, mainly due to increase in MPI, is well reproduced by POWHEG+PYTHIA8.
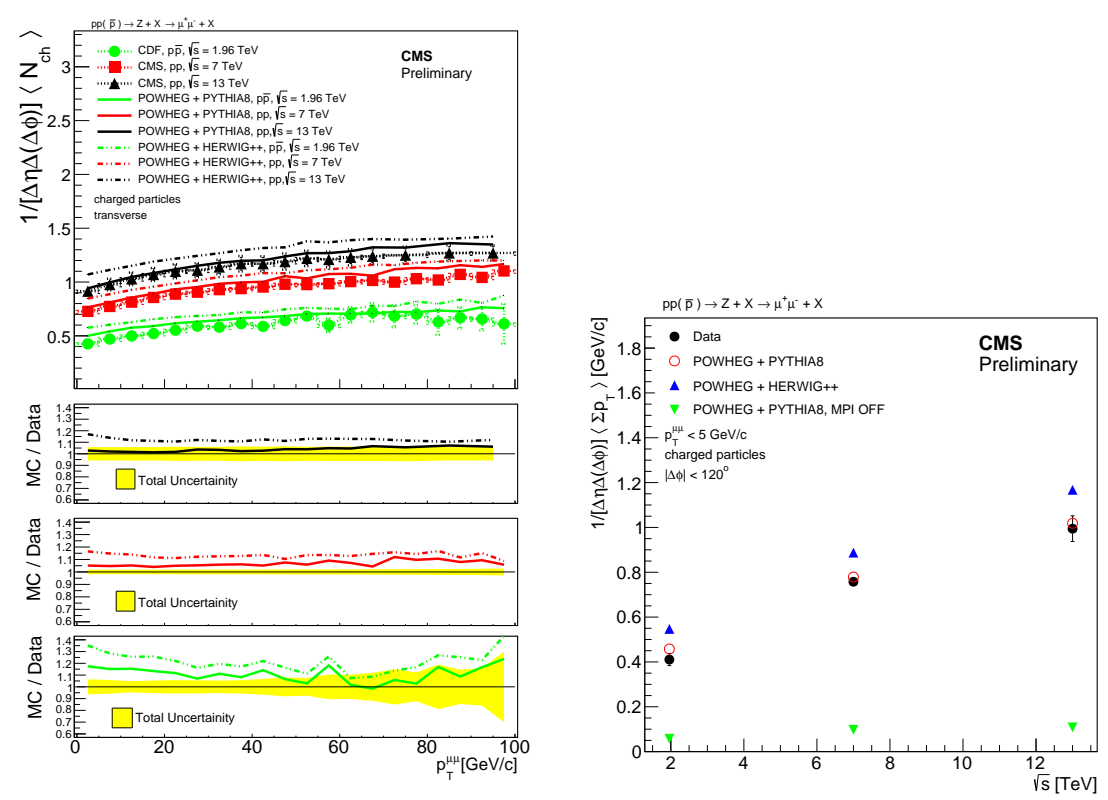

Figure 1: Comparison of UE activity measured at different center-of-mass energies with MC predictions for the particle density in the transverse region (left). The bottom panels of the plot report the ratios of model predictions to the data, with error band representing the quadrature sum of statistical and systematic uncertainties. Comparison of $\sum \mathrm{p}_{\mathrm{T}}$ as a function of $\sqrt{\mathrm{s}}$ for data, and predictions from simulations (right). The error bars represent the statistical and systematic uncertainties added in quadrature [2].

For the $t \bar{t}$ events, the modeling of the initial-and final-state-radiations in simulations is investigated using the distributions of $\mathrm{N}_{\mathrm{ch}}$ and $\sum \mathrm{p}_{\mathrm{T}}^{\mathrm{ch}}$ for different values of parton showering scale (Q) [3], in phase space regions defined based on $\Delta \phi$ between the charged particle and the $\bar{t} \bar{t}$ system. Figure 2 (left) illustrates the detector-level distribution of $\mathrm{N}_{\mathrm{ch}}$ with $\mathrm{Q}$ increased from its default value. The predictions from the POWHEGV2+PYTHIA 8 agree well with data in all regions, though a slightly better agreement is observed for charged multiplicities $\leq 50$. The parton showering scale dependence is more prominent when the UE activity is measured as a function of $\mathrm{p}_{\mathrm{T}}$ of the $\overline{\mathrm{t}} \operatorname{system}\left(\mathrm{p}_{\mathrm{T}}^{\mathbf{t}}\right)$ and is shown in Fig. 2 (right) using the $\sum \mathrm{p}_{\mathrm{T}}$ variable. The distributions obtained with the increased scale, $(2 \mathrm{Q})^{2}$, matches better with the data. An increase in $\sum \mathrm{p}_{\mathrm{T}}$ is observed in the away region with increasing $\left(\mathrm{p}_{\mathrm{T}}^{\mathrm{t}}\right)$, due to the increased jet activity.

\section{Double parton scattering}

The DPS cross section is measured using the same-sign $\mathrm{W}$ boson pair production at $\sqrt{\mathrm{s}}=13$ $\mathrm{TeV}$ [4]. The leptonic decay of two $\mathrm{W}$ bosons is considered into a pair of muons or an electronmuon pair, resulting in experimentally clean final states. A multivariate analysis based on boosted decision trees (BDT) is used to discriminate the signal against the background processes. The BDTs are trained using a set of input variables which are defined based on the topological differences between the DPS signal and background processes. To maximize the sensitivity of the signal 

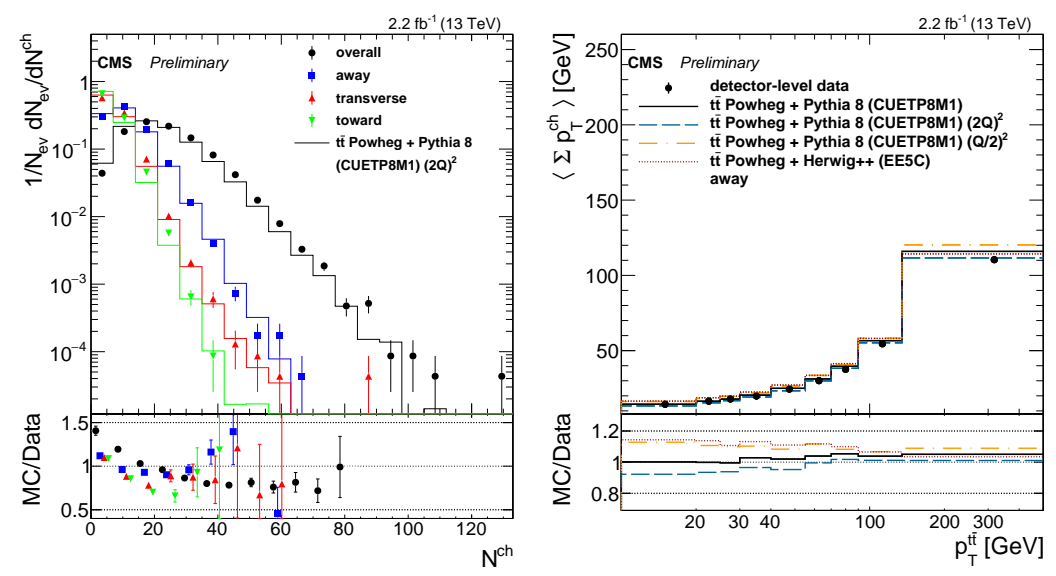

Figure 2: Detector-level distribution of $\mathrm{N}_{\mathrm{ch}}$ with increased value of $\mathrm{Q}^{2}$ for the away, transverse, and toward regions as well as for the overall sample (left). The points correspond to the data and the lines represent thePOWHEGV2+PYTHIA8 predictions with the CUETP8M1 tune. The CMS data at $13 \mathrm{TeV}$ on the normalized cross section in the lepton+jets channel. On the right plot, distribution of $\sum \mathrm{p}_{\mathrm{T}}^{\mathrm{ch}}$ versus $\mathrm{p}_{\mathrm{T}}^{\mathrm{t}}$ in the away region is shown. The data distributions at the detector level are compared with the POWHEGV2+PYTHIA8 predictions with the CUETP8M1 tune generated with different values of $\mathrm{Q}^{2}$, and with the POWHEG+HERWIG++ sample with the EE5C tune [3].

process, the statistical analysis is performed by categorizing the shape of the BDT discriminant into different charge configurations of the final state leptons. The obtained results are also compared with predictions from the PYTHIA8 and those obtained using the factorization approach [5] with a $\sigma_{\text {eff }}$ of $20.7 \pm 6.6 \mathrm{mb}$ as measured in $\mathrm{W}+2$ jets final state at $7 \mathrm{TeV}$ [6]. The measurement of DPS cross section is found to be compatible with both the predictions at the $95 \%$ confidence level and is used to extract $\sigma_{\text {eff. }}$. Figure 3 shows the comparison of obtained value of $\sigma_{\text {eff }}$ with previous measurements at different center-of-mass energies. All the measurements are compatible within large systematic uncertainties and hence it is not possible to draw conclusions on the process and collision energy dependence of $\sigma_{\text {eff }}$.

\section{Conclusions}

This note presents the recent measurements from the CMS Collaboration on the underlying event (UE) activity and double parton scattering (DPS), using the proton-proton collision data at a center-of-mass energy of $13 \mathrm{TeV}$. The UE activity is measured using inclusive $\mathrm{Z}$ boson and $t \bar{t}$ production, while the DPS cross section measurement is performed using the same-sign WW events. For the $\mathrm{Z}$ boson events, the distributions are corrected for detector effects and compared with various predictions and the results are also compared with previous measurements at 1.96 and $7 \mathrm{TeV}$. The increase in the UE activity with the increase in collision energy from 7 to $13 \mathrm{TeV}$ is nicely described by the simulations. But, the increase in UE activity from 1.96 to $13 \mathrm{TeV}$ (by a factor of $\sim 2$ ) is underestimated by the predictions, which further demands the improvements in the UE modeling in th Monte Carlo models. For the $t \bar{t}$ events, the detector-level data distributions are compared with the predictions from POWHEG interfaced with PYTHIA8 and HERWIG++. Both 


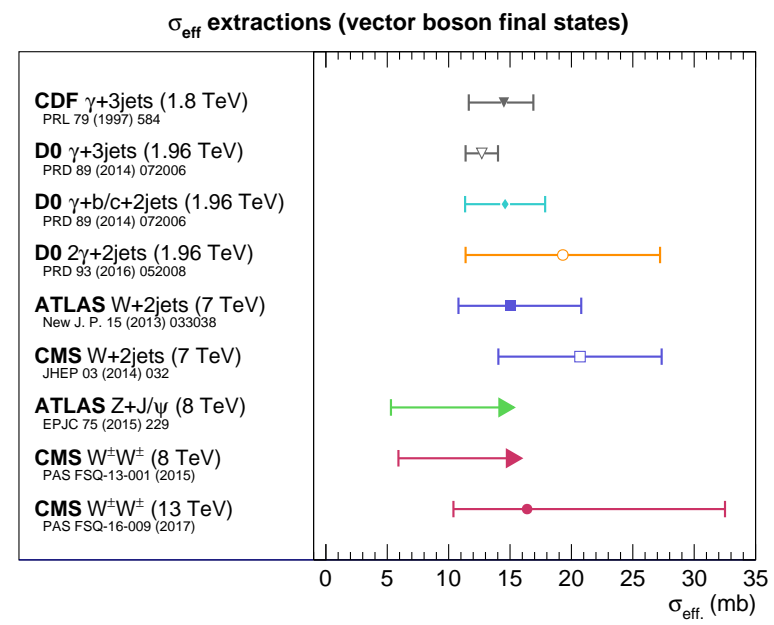

Figure 3: $\sigma_{\text {eff }}$ measurements from different experiments at different center-of-mass energies. The data points are taken from the references quoted in the figure.

the predictions describe the data well, including the UE-sensitive observables. The UE-sensitive observables are found to be sensitive towards parton shower scale variations, when measured as a function of $\mathrm{p}_{\mathrm{T}}$ of $\mathrm{t} \overline{\mathrm{t}}$ system. Such measurements of UE activity in t⿱亠t events at particle-level might lead to more precise top quark mass measurements with better understood systematic uncertainties. For the DPS cross section measurements, the leptonic decay of same-sign $\mathrm{W}$ boson pair offers a experimentally clean signature. A set of DPS-sensitive observables are used to train boosted decision trees (BDT). The shape of the BDT discriminant is used to perform the statistical analysis which yields the value of cross section for WW production via DPS ( $\left.\sigma_{\mathrm{DPS}}\right)$. The extracted value of $\sigma_{\mathrm{DPS}}$ is used to calculate effective cross section, whose value is found to be compatible with the already existing values at different center-of-mass energies.

\section{References}

[1] CMS Collaboration, “The CMS experiment at the CERN LHC”, JINST 3 S08004 (2008), doi:10.1088/1748-0221/3/08/S08004.

[2] CMS Collaboration,"Measurement of the underlying event using the Drell-Yan process in proton-proton collisions at $\sqrt{\mathrm{s}}=13 \mathrm{TeV}$ ", CMS-PAS-FSQ-16-008 (2016)

https://cds.cern.ch/record/2238518.

[3] CMS Collaboration,"Underlying event measurement with tbart+X events with p-p collision data at $\sqrt{\mathrm{s}}=13$ TeV", CMS-PAS-TOP-15-017 (2015) https: //cds. cern.ch/record/2114809.

[4] CMS Collaboration,"Measurement of double parton scattering in same-sign WW production in p-p collisions at $\sqrt{\mathrm{s}}=13 \mathrm{TeV}$ with the CMS experiment", CMS-PAS-FSQ-16-009 (2017) https://cds.cern.ch/record/2257583.

[5] J.R. Gaunt et al., "Same-sign W pair production as a probe of double parton scattering at the LHC", EPJC 6953 (2010) doi:10.1140/epjc/s10052-010-1362-y

[6] CMS Collaboration, "Study of double parton scattering using $\mathrm{W}+2$-jet events in proton-proton collisions at $\sqrt{\mathrm{s}}=7$ TeV", JHEP 1403032 (2014) doi:10.1007/JHEP03 (2014) 032. 